PROCEEDINGS OF THE

AMERICAN MATHEMATICAL SOCIETY

Volume 134, Number 1, Pages 99-104

S 0002-9939(05)07886-X

Article electronically published on August 22, 2005

\title{
LINEAR FUNCTIONALS ON THE CUNTZ ALGEBRA
}

\author{
EUI-CHAI JEONG
}

(Communicated by David R. Larson)

\begin{abstract}
For a pure state $p^{\prime}$ on $\mathcal{O}_{n}$, which is an extension of a pure state $p$ on $\mathrm{UHF}_{n}$ with the property that if $\left(\mathcal{H}_{p^{\prime}}, \pi_{p^{\prime}}, \omega_{p^{\prime}}\right)$ is a corresponding representation, then $\pi_{p^{\prime}}\left(\mathrm{UHF}_{n}\right)=B\left(\mathcal{H}_{p^{\prime}}\right), p^{\prime}$ induces a unital shift of $B(\mathcal{H})$ of the Powers index $n$. We describe states $p$ on $\mathrm{UHF}_{n}$ by using sequences of unit vectors in $\mathbb{C}^{n}$. We study the linear functionals on the Cuntz algebra $\mathcal{O}_{n}$ whose restrictions are the product pure state on $\mathrm{UHF}_{n}$. We find conditions on the sequence of unit vectors for which the corresponding linear functionals on $\mathcal{O}_{n}$ become states under these conditions.
\end{abstract}

\section{IntRoduction}

A non-degenerate representation of the Cuntz algebra $\mathcal{O}_{n}$ induces a unital endomorphism of $B(\mathcal{H})$ of Powers index $n$. We consider the uniformly hyperfinite algebra $\mathrm{UHF}_{n}$ as a subalgebra of the Cuntz algebra $\mathcal{O}_{n}$. For a representation $\pi$ of the Cuntz algebra $\mathcal{O}_{n}, \pi\left(\mathrm{UHF}_{n}\right)$ is weakly dense in $B(\mathcal{H})$ if and only if the corresponding unital endomorphism of $B(\mathcal{H})$ is a shift of Powers index $n$ [BJP. By GNS construction, a study of the representation of the $C^{*}$-algebra $\mathcal{O}_{n}$ is equivalent to a study of its state. Let $\mathcal{H}$ be a separable infinite dimensional Hilbert space and denote by $B(\mathcal{H})$ the von Neumann algebra of all linear operators on $\mathcal{H}$. The term endomorphism will denote a $*$-homomorphism of $B(\mathcal{H})$ into itself. The study of endomorphisms of the von Neumann algebras, especially of $B(\mathcal{H})$, has received increased attention in connection with several related areas such as the Jones index for subfactor duality for compact groups [Jon, Wor. Let $\varphi: B(\mathcal{H}) \rightarrow B(\mathcal{H})$ be a unital endomorphism. The Powers index $n \in\{1,2, \cdots, \infty\}$ of $\varphi$ is defined by $n$ such that $\varphi(B(\mathcal{H}))^{\prime} \cap B(\mathcal{H})$ is isomorphic to the factor of type $I_{n}$ Pow. A unital endomorphism $\varphi: B(\mathcal{H}) \rightarrow B(\mathcal{H})$ is said to be a shift if $\bigcap_{k=1}^{\infty} \varphi^{k}(B(\mathcal{H}))=\mathbb{C} 1$. In BJP] and [Lac, the authors study the program initiated by Powers of analyzing the conjugacy classes of discrete shifts on $B(\mathcal{H})$. The main theme is to describe the correspondence between endomorphisms of Powers index $n \in\{1,2, \cdots, \infty\}$ and representations of the Cuntz algebra $\mathcal{O}_{n}$ which implement the endomorphisms. Theorem 1.1 in [BJP] and Theorem 4.5 in [Lac show a characterization of the conjugacy study that is focused on the pure states on $\mathrm{UHF}_{n}$ and their extensions on $\mathcal{O}_{n}$ arising in the study of the correspondence between endomorphisms, especially the shift of Powers index $n$ of $B(\mathcal{H})$, and as representations of $\mathcal{O}_{n}$ as described above. We restrict $n$ as finite throughout this paper.

Received by the editors October 25, 2000.

2000 Mathematics Subject Classification. Primary 46L05; Secondary 46L40.

This work was supported by the Brain Korea 21 Project. 
Definition 1.1 ([ $\mathrm{Pow}]$ ). A uniformly hyperfinite algebra UHF is a $C^{*}$-algebra which is the norm closure of an increasing sequence of type $I_{n_{i}}$-factors that can be identified with $\bigotimes_{i=1}^{\infty} M_{n_{i}}$. A $\mathrm{UHF}_{n}$ algebra is the UHF algebra Glimm type $n^{\infty}$, that is, $\otimes M_{n}$.

Definition 1.2 ( $\mathrm{Cun}$ ). The Cuntz algebra $\mathcal{O}_{n}, n=2,3, \ldots$, is the universal $C^{*}$-algebra generated by $n$ isometries $s_{1}, s_{2}, \cdots, s_{n}$ subject to the relations

$$
s_{i}^{*} s_{j}=\delta_{i j} 1 \quad \text { and } \quad \sum_{i=1}^{n} s_{i} s_{i}^{*}=1 .
$$

In this paper, we consider $\mathrm{UHF}_{n}$ as a subalgebra of $\mathcal{O}_{n}$. That is, $\mathrm{UHF}_{n}$ is the closure of the linear span of all Wick-ordered monomials of the form

$$
s_{i_{1}} s_{i_{2}} \cdots s_{i_{k}} s_{j_{k}}^{*} s_{j_{k-1}}^{*} \cdots s_{j_{1}}^{*}
$$

over $k=0,1,2, \ldots$.

Theorem 1.3 (Theorem 2.1 in [Arv, Proposition 2.2 in Lac, and Theorem 3.1 in $[\mathrm{BJP}])$. Let $\varphi$ be a unital endomorphism of $B(\mathcal{H})$ of Powers index $n=2,3, \ldots$ It follows that there exists a non-degenerate representation of $\mathcal{O}_{n}$ on $\mathcal{H}$ such that

$$
\varphi(X)=\sum_{i=1}^{n} S_{i} X S_{i}^{*}
$$

where $S_{i}$ is the representative of $s_{i}$. Conversely, any non-degenerate representation of $\mathcal{O}_{n}$ on $\mathcal{H}$ defines an endomorphism of index $n$ by (1.3). The representation is unique up to the canonical action of the unitary group $\mathcal{U}(n)$.

A canonical representation of the $n$-dimensional unitary group $\mathcal{U}(n)$ in the automorphism group of $\mathcal{O}_{n}$ is defined by $\mathcal{T}_{g}\left(s_{i}\right)=\sum_{j=1}^{n} g_{j i} s_{j}$ for $g=\left[g_{i j}\right]_{i, j=1}^{n} \in \mathcal{U}(n)$. The canonical action of $\mathcal{U}(n)$ on $\mathcal{O}_{n}$ gives rise to an action of $\mathcal{U}(n)$ on each of these spaces. Also, the unitary group $\mathcal{U}(n)$ on $\mathcal{H}$ acts on each of these spaces where $U \in U(\mathcal{H})$, and $\pi$ is the map

$$
\pi(\cdot) \rightarrow U \pi(\cdot) U *
$$

for non-degenerate representations of $\mathcal{O}_{n}$ on $\mathcal{H}$. Details are in BJP.

Theorem 1.4 (Theorem 3.3 in BJP). Let $\pi \rightarrow \varphi(\pi)$ be the surjective map from the set of non-degenerate representations of $\mathcal{O}_{n}$ on $\mathcal{H}$ onto the set of unital endomorphisms of $B(\mathcal{H})$ of Powers index $n$. Then $\pi\left(\mathrm{UHF}_{n}\right)$ is weakly dense in $B(\mathcal{H})$ if and only if $\varphi(\pi)$ is a unital shift on $B(\mathcal{H})$ of Powers index $n$. These representations identify with the cycle representation defined by any vector state, defined by a unit vector in $\mathcal{H}$. The corresponding states on $\mathcal{O}_{n}$ can be characterized abstractly by the restriction of those states to $\mathrm{UHF}_{n}$. Let $P$ denote the set of pure states $p$ on $\mathrm{UHF}_{n}$ such that it has a pure extension $p^{\prime}$ to $\mathcal{O}_{n}$ with the property that, if $\left(\mathcal{H}_{p^{\prime}}, \pi_{p^{\prime}}, \Omega_{p^{\prime}}\right)$ is the corresponding representation, then $\pi_{p^{\prime}}\left(\mathrm{UHF}_{n}\right)^{\prime \prime}=B\left(\mathcal{H}_{p^{\prime}}\right)$.

\section{An extension on $\mathcal{O}_{n}$ OF the pure state on $\mathrm{UHF}_{n}$}

With the identification $\mathrm{UHF}_{n}$ as an infinite tensor product $\otimes M_{n}$, an element

$$
s_{i_{1}} \cdots s_{i_{k}} s_{j_{k}}^{*} \cdots s_{j_{1}}^{*} \in \mathrm{UHF}_{n} \subset \mathcal{O}_{n}
$$

can be identified with

$$
E_{i_{1} j_{1}} \otimes \cdots \otimes E_{i_{1} j_{1}} \otimes I \otimes I \otimes \cdots
$$


where $E_{i j}$ is the matrix in $M_{n}$ whose $(i, j)$-element is 1 and the others are 0 . Let $P$ be the set of pure states $p$ on $\mathrm{UHF}_{n}$ which has a pure state extension $p^{\prime}$ to $\mathcal{O}_{n}$ with the property that if $\left(\mathcal{H}_{p^{\prime}}, \pi_{p^{\prime}}, \Omega_{p^{\prime}}\right)$ is the corresponding representation, then $\pi_{p^{\prime}}\left(\mathrm{UHF}_{n}\right)^{\prime \prime}=B\left(\mathcal{H}_{p^{\prime}}\right)$. Let $p_{i}=\left.p\right|_{M_{n_{i}}}$, where $M_{n_{i}}$ is the $i$-th $M_{n}$ in the infinite tensor product $\otimes M_{n}$. Then $p_{i}$ is a pure state on $M_{n}$. Since $p=\bigotimes_{i=1}^{\infty} p_{i}$, the pure state $p$ is a product pure state. We are going to study pure states $p$ on $\mathrm{UHF}_{n}$ for a $p_{i}$ vector state on $\mathbb{C}^{n}$ and find their pure extension $p^{\prime}$ on $\mathcal{O}_{n}$.

Definition 2.1. Let $a_{m}=\left(a_{m}^{1}, \ldots, a_{m}^{n}\right)$ be a unit vector in $\mathbb{C}^{n}$ and let $\left\{a_{m}\right\}_{m=1}^{\infty}$ be a sequence of unit vectors. A pure state $\mu_{a_{m}}$ on $\mathrm{UHF}_{n}$ is defined by

$$
\mu_{a_{m}}\left(E_{i_{1} j_{1}} \otimes E_{i_{2} j_{2}} \otimes \cdots \otimes E_{i_{l} j_{l}} \otimes I \otimes \cdots\right)=a_{1}^{i_{1}} a_{1}^{\bar{j}_{1}} a_{2}^{i_{2}} a_{2}^{\bar{j}_{1}} \cdots a_{l}^{i_{l}} a_{2}^{\bar{j}_{l}}
$$

for $D_{i_{1} j_{1}} \otimes E_{i_{2} j_{2}} \otimes \cdots \otimes E_{i_{l} j_{l}} \otimes I \otimes \cdots \in \mathrm{UHF}_{n}$.

Definition 2.2. For a sequence $\left\{a_{m}\right\}_{m=1}^{\infty}$ of unit vectors in $\mathbb{C}^{n}$, the linear functional $\mu_{a_{m}}^{\prime}$ on the Cuntz algebra $\mathcal{O}_{n}$ is defined by

$$
\mu_{a_{m}}^{\prime}\left(s_{i_{1}} \cdots s_{i_{l}} s_{i_{k}}^{*} \cdots s_{j_{1}}^{*}\right)=a_{1}^{i_{1}} \cdots a_{l}^{i_{l}} a_{k}^{\bar{j}_{k}} \cdots a_{2}^{\bar{j}_{l}} .
$$

Since $\mu_{a_{m}}^{\prime} \mid \mathrm{UHF}_{n}=\mu_{a_{m}}$ is a pure state on $\mathrm{UHF}_{n}, \mu_{a_{m}}^{\prime} \in P$ if $\mu_{a_{m}}^{\prime}$ is a pure state on $\mathcal{O}_{n}$. The linear functional $\mu_{a_{m}}^{\prime}$ on $\mathcal{O}_{n}$ coming from (2.2) is not a state in general, but a linear functional on $\mathcal{O}_{n}$.

The following is an example of this.

Example 2.3. Let $n=2, a_{1}=(-1,0)$ and $a_{n}=(1,0)$ for all $m=2,3, \ldots$ If $\sqrt{3} x=s_{1}^{*}\left(1+s_{1}+s_{1} s_{1}\right)$, then

$$
\begin{aligned}
\mu_{a_{m}}^{\prime}\left(x^{*} x\right) & =\frac{1}{3} \mu_{a_{m}}^{\prime}\left(2+2 s_{1}+2 s_{1}^{*}+s_{1} s_{1}+s_{1} s_{1}^{*}+s_{1}^{*} s_{1}^{*}\right) \\
& =\frac{1}{3}\{2+2(-1)+2(-1)+(-1) 1+(-1)(-1)+(-1)(1)\} \\
& =-1 .
\end{aligned}
$$

If $a \sim b$ means $a=\lambda b$ for some $\lambda \in \mathbb{C}$ with $|\lambda|=1$, then $\sim$ is an equivalence relation on $\mathbb{C}$. Let $\mathbf{a}$ be a representative of an equivalence class of $a$.

Theorem 2.4. There is a one-to-one correspondence between the set of sequences $\left\{\mathbf{a}_{m}\right\}_{m}$ for unit vectors $a_{m} \in \mathbb{C}^{n}$ and the set of product pure states on the $\mathrm{UHF}_{n}$ algebra.

Proof. If we set $p_{i}(x)=\left\langle x a_{m}, a_{m}\right\rangle$, then the proof follows straightforwardly with $p(\cdot)=\otimes\left\langle\cdot a_{m}, a_{m}\right\rangle$ and $\left\langle\cdot a_{m}, a_{m}\right\rangle=\left\langle\cdot \lambda a_{m}, \lambda a_{m}\right\rangle$ for $|\lambda|=1$.

\section{Conditions of $\left\{a_{m}\right\}$ to be a state on $\mathcal{O}_{n}$}

As we showed in Example 2.3, the linear functional on $\mathcal{O}_{n}$ defined in (2.2) is not a state on $\mathcal{O}_{n}$ in general. However, the state $\left.\mu_{\left\{a_{n}\right\}}\right|_{\mathrm{UHF}_{n}}=\mu_{a_{m}}$ on $\mathrm{UHF}_{n}$ always has a pure state extension on $\mathcal{O}_{n}$ [BJP. A pure state extension of the product pure state $\mu_{a_{m}}$ on $\mathrm{UHF}_{n}$ may differ from the one defined in (2.2). We first look for conditions of the sequence $\left\{a_{m}\right\}$ so that the linear functional $\mu_{\left\{a_{m}\right\}}^{\prime}$ becomes a pure state on the Cuntz algebra $\mathcal{O}_{n}$.

Theorem 3.1. For the state $\mu$ on $\mathcal{O}_{n}, \mu\left(s_{i} s_{i}^{*}\right)=\mu\left(s_{i}\right) \mu\left(s_{i}^{*}\right)$ if $\sum_{i=1}^{n}\left|\mu\left(s_{i}\right)\right|^{2}=1$. 
Proof. Let $N=\left(\begin{array}{c}\mu\left(s_{1}^{*}\right) \cdots \mu\left(s_{n}\right)^{*} \\ 0 \cdots 0 \\ 0 \cdots 0\end{array}\right)$ be an $n \times n$ matrix in $M_{n}\left(\mathcal{O}_{n}\right)$. For two unit vectors $a$ and $b$ in $\mathbb{C}^{n}$, let $c$ and $d$ be the real numbers satisfying

$$
a N b=c e^{i d} .
$$

For any real number $x$, the quadratic equation in $x$,

$$
\begin{aligned}
x^{2}+ & 2 c x+a \\
& =\left(x e^{i d} a, b\right)\left(\begin{array}{ccccccc}
1 & & & 0 & \mu\left(s_{1}^{*}\right) & \cdots & \mu\left(s_{n}^{*}\right) \\
& \ddots & & & 0 & \cdots & 0 \\
0 & & & 1 & 0 & \cdots & 0 \\
\mu\left(s_{1}\right) & 0 & \cdots & 0 & \mu\left(s_{1} s_{1}^{*}\right) & \cdots & \mu\left(s_{1} s_{n}^{*}\right) \\
\ddots & & & & \ddots & & \ddots \\
\mu\left(s_{n}\right) & 0 & \cdots & 0 & \mu\left(s_{n} s_{1}^{*}\right) & \cdots & \mu\left(s_{n} s_{n}^{*}\right)
\end{array}\right)\left(x e^{i d} a, b\right)^{*} \\
& =\left(x e^{i d} a, b\right)\left(\begin{array}{cc}
I_{n} & N \\
0 & 0
\end{array}\right)^{*}\left(\begin{array}{cc}
I_{n} & N \\
0 &
\end{array}\right)\left(x e^{i d} a, b\right)^{*},
\end{aligned}
$$

is non-negative with $\alpha=b\left(\mu\left(s_{i} s_{j}^{*}\right)\right)_{i, j=1}^{n} b^{*}$ and $c=e^{i d} a N b$. Since $x^{2}+2 c x+\alpha \geq 0$, we have $D=4 C^{2}-4 \alpha \leq 0$. Then

$$
|c|^{2}=\left|e^{i d} a N b\right|^{2} \leq b\left(\mu\left(s_{i} s_{j}^{*}\right)\right) b^{*}=\alpha,
$$

and

$$
|\langle a N, b\rangle|^{2} \leq\left\langle b\left(\mu\left(s_{i} s_{j}^{*}\right)\right), b\right\rangle .
$$

When $a N \neq 0$, if we replace $a$ by the normalized unit vector $\frac{b N^{*}}{\|b N *\|}$, then

$$
\left\langle b N^{*} N, b\right\rangle \leq\left\langle b\left(\mu\left(s_{1} s_{j}^{*}\right)\right), b\right\rangle .
$$

We have $N^{*} N \leq \mu\left(s_{i} s_{j}^{*}\right)$ which implies

$$
\left(\begin{array}{cccc}
\mu\left(\bar{s}_{1}^{*}\right) & 0 & \cdots & 0 \\
\cdots & & & \\
\mu\left(\bar{s}_{n}^{*}\right) & 0 & \ddots & 0
\end{array}\right)\left(\begin{array}{ccc}
\mu\left(s_{1}^{*}\right) & \cdots & 0 \\
0 & \cdots & \\
\ddots & & \ddots \\
0 & \cdots & 0
\end{array}\right) \leq\left(\begin{array}{cccc}
\mu\left(s_{1} s_{1}^{*}\right) & \cdots & \mu\left(s_{1} s_{n}^{*}\right) \\
\ddots & & \ddots \\
\mu\left(s_{n} s_{1}^{*}\right) & \cdots & \mu\left(s_{n} s_{n}^{*}\right)
\end{array}\right) .
$$

Thus

$$
\mu\left(s_{i}\right) \mu\left(\bar{s}_{j}\right) \leq \mu\left(s_{i} s_{j}^{*}\right)
$$

Now,

$$
\begin{aligned}
\sum_{i=1}^{n}\left|\mu\left(s_{i}\right)\right|^{2} & =\operatorname{tr}\left(\mu\left(s_{i}\right) \mu\left(\bar{s}_{j}\right)\right) \leq \operatorname{tr}\left(\mu\left(s_{i} s_{j}^{*}\right)\right) \\
& =\sum_{i=1}^{n} \mu\left(s_{i} s_{i}^{*}\right) \\
& =\mu \sum_{i=1}^{n}\left(s_{i} s_{i}^{*}\right) \\
& =1
\end{aligned}
$$


If $\sum_{i=1}^{n}\left|\mu\left(s_{i}\right)\right|^{2}=1$, then $\left(\mu\left(s_{i}\right) \mu\left(\bar{s}_{j}\right)-\mu\left(s_{i} s_{j}^{*}\right)\right)$ is a positive matrix with its trace 0 . Thus $\left(\mu\left(s_{i}\right) \mu\left(\bar{s}_{j}\right)-\mu\left(s_{i} s_{j}^{*}\right)\right)$ is a zero matrix. Hence we have

$$
\left(\mu\left(s_{i}\right) \mu\left(\bar{s}_{j}\right)\right)=\left(\mu\left(s_{i} s_{j}^{*}\right)\right)
$$

for $i, j-1, \ldots, n$.

Theorem 3.2. If $\left(\mu_{1}\left(s_{1}\right), \ldots, \mu\left(s_{n}\right)\right)$ and $\left(\mu_{2}\left(s_{1}\right), \ldots, \mu_{2}\left(s_{n}\right)\right)$ are unit vectors in $\mathbb{C}^{n}$ for states $\mu_{1}$ and $\mu_{2}$ on $\mathcal{O}_{n}$ satisfying $\left.\mu_{1}\right|_{\mathrm{UHF}_{n}}=\left.\mu_{2}\right|_{\mathrm{UHF}_{n}}$, then there exists a complex number $\lambda,|\lambda|=1$, such that

$$
\begin{aligned}
\left(\mu_{1}\left(s_{1}\right), \ldots, \mu_{1}\left(s_{n}\right)\right) & =\lambda\left(\mu_{2}\left(s_{1}\right), \ldots, \mu_{2}\left(s_{n}\right)\right) \\
& =1 .
\end{aligned}
$$

Proof. Since $s_{i} s_{i}^{*} \in \mathrm{UHF}_{n}$, we have $\mu_{1}\left(s_{i} s_{i}^{*}\right)=\mu_{2}\left(s_{i} s_{i}^{*}\right)$ for $i=1, \ldots, n$, and

$$
\begin{aligned}
& \left(\mu_{1}\left(s_{1}\right), \ldots, \mu_{1}\left(s_{n}\right)\right)\left(\mu_{1}\left(s_{1}\right), \ldots, \mu_{1}\left(s_{n}\right)\right)^{*} \\
& \quad=\left(\mu_{2}\left(s_{1}\right), \ldots, \mu_{2}\left(s_{n}\right)\right)\left(\mu_{2}\left(s_{1}\right), \ldots, \mu_{2}\left(s_{n}\right)\right)^{*}
\end{aligned}
$$

by Theorem 3.1. Note that

$$
\left(\mu_{1}\left(s_{1}\right), \ldots, \mu_{1}\left(s_{n}\right)\right)^{*}\left(\mu_{1}\left(s_{1}\right), \ldots, \mu_{1}\left(s_{n}\right)\right)
$$

and

$$
\left(\mu_{2}\left(s_{1}\right), \ldots, \mu_{2}\left(s_{n}\right)\right)^{*}\left(\mu_{2}\left(s_{1}\right), \ldots, \mu_{2}\left(s_{n}\right)\right)
$$

are projections of rank 1 and their eigenvectors are $\left(\mu_{1}\left(s_{1}\right), \ldots, \mu_{1}\left(s_{n}\right)\right)$ and $\left(\mu_{2}\left(s_{1}\right), \ldots, \mu_{2}\left(s_{n}\right)\right)$, respectively. Since the eigenspace dimension is one, two unit vectors are linear dependent. Hence, for some complex number $\lambda$ such that $|\lambda|=1$, we have $\left(\mu\left(s_{1}\right), \ldots, \mu_{1}\left(s_{n}\right)\right)=\lambda\left(\mu_{2}\left(s_{1}\right), \ldots, \mu_{2}\left(s_{n}\right)\right)$.

Theorem 3.3. Let $a_{m}=\lambda_{m} a$ for unit vectors $a, a_{m}$, and $\lambda_{m}$ in $\mathbb{C}$ for $m \in \mathbb{N}$. If $\mu_{a_{m}}$ is a state on $\mathcal{O}_{n}$, then $\lambda_{m}=1$ for $m \in \mathbb{N}$.

Proof. Let $\varphi(x)=\sum_{i=1}^{n} s_{i} x s_{i}^{*}$ for $x \in \mathcal{O}_{n}$. For state $\mu \in \mathcal{O}_{n}$,

$$
\begin{aligned}
& \mu\left(\sum_{i=1}^{n}\left(\varphi^{k-1}\left(s_{i}\right)-\varphi^{k}\left(s_{i}\right)\right)\left(\varphi^{k-1}\left(s_{i}\right)-\varphi^{k}\left(s_{i}\right)\right)^{*}\right) \\
& \quad=\mu\left(\sum_{i=1}^{n}\left(\varphi^{k-1}\left(s_{i} s_{i}^{*}\right)+\varphi^{k}\left(s_{i} s_{i}^{*}\right)-\varphi^{k-1}\left(\varphi\left(s_{i}\right) s_{i}^{*}\right)-\varphi^{k-1}\left(s_{i} \varphi\left(s_{i}^{*}\right)\right)\right)\right)^{*} \\
& \quad=\mu(1+1)-2 \varphi^{k-1}\left(\sum_{i, j=1}^{n} s_{j} s_{i} s_{i}^{*} s_{i}\right) \\
& \quad=0 .
\end{aligned}
$$

Thus we have

$$
\mu\left(\varphi^{k-1}\left(s_{i}\right)\right)=\mu\left(\varphi^{k}\left(s_{i}\right)\right)
$$

for $i=1, \ldots, n$. If we set $\mu=\mu_{a_{m}}$, then $a_{m}^{i}=a_{m+1}^{i}$. Hence we have $\lambda_{m}=1$ for $m \in \mathbb{N}$.

According to Theorem 3.3, the linear functional $\mu_{a_{m}}$ defined in (2.2) is a state only for the constant sequence $a_{m}=a$ for the unit vector $a_{m}$ for all $m=1,2, \ldots$. This type of linear functional appeared in [BJP]. Theorem 3.3 is proof of the positivity, which implies that it is a pure state on $\mathcal{O}_{n}$. 


\section{REFERENCES}

[Arv] W. B. Arveson, Continuous analogues of Fock space I, Mem. Amer. Math. Soc. 80, no. 409. MR0987590 (90f:47061)

[BrJo] O. Bratteli, P. E. T. Jorgensen, Isometries, shifts, Cuntz algebras and multiresolution wavelet analysis of scale N, Integral Equations Operator Theory 28 (1997), 382-432. MR.1465320 (99k:46094b)

[BJP] O. Bratteli, P. E. T. Jorgensen, and G. L. Price, Endomorphisms of B(H), Proc. Symposi. Pure Math. 59 (1996), 93-138. MR1392986 (97h:46107)

[Cun] J. Cuntz, Simple $C^{*}$-algebras generated by isometries, Commun. Math. Phys. 57 (1977), 173-185. MR0467330 (57:7189)

[Lac] M. Laca, Endomorphisms of B(H) and Cuntz algebras, J. Oper. Theory 30 (1993), 85-108. MR.1302609 (95m:46100)

[Jon] V. F. R. Jones, Hecke algebra representations of braid groups and link polynomials, Ann. Math. 126 (1987), 335-388. MR0908150 (89c:46092)

[Pow] R. T. Powers, An index theory for semigroups of $*$-endomorphisms of $B(\mathcal{H})$ and type $I I_{1}$ factors, Canad. J. Math. 40 (1988), 46-114. MR0928215 (89f:46116)

[Wor] S. L. Woronowicz, Compact matrix pseudogroups, Commun. Math. Phys. 111 (1987), 613655. MR0901157 (88m:46079)

Department of Mathematics, Chung-Ang University, Dongjak-ku, Seoul, 156-756, SOUTH Korea

E-mail address: jeong@cau.ac.kr 